\title{
A Model Application of Two Phase Commit Protocol in Wireless DTN
}

\author{
G. U. Mali \\ Electronics and \\ Telecommunication Department \\ Vasantrao More Polytechnic \\ Tehu, Parola Maharashtra, India
}

\author{
D. K. Gautam \\ Department of \\ Electronics Engg. and Tech. \\ North Maharashtra University, \\ Jalgaon \\ Maharashtra, India
}

\begin{abstract}
The requirements of high band width data spectrum and its efficient high speed transmission is the challenge which is exponentially rising due to the automation of all kinds of electrical and electronic gadgets. Currently most of the local area networks are adhoc due to which connectivity is a serious problem. The currently used DTN has several problems of call drop and data failure. Recently two phase commit protocol has been proposed which has high potential to overcome the problems of call drop. Two phase commit protocol on deployment in real time scenario has been analyzed for the DTN networks and we found most astonishing results in the improvement of the routing paradigm as compared to DSR and AODV protocols for routing throughput and for average end to end delay. This paper analyzes two phase commit protocol running in two different phases on deploying the DTN wireless scenario for heterogeneous network infrastructure and measures the effects successfully.
\end{abstract}

\section{Keywords}

Two phase commit protocol, Disruption tolerant network, Wireless network, Hop.

\section{INTRODUCTION}

The rapid increase in online electronic gadgets exponentially enhances the demand of highly efficient Internet and intranet network in the current era of digital industry. Nowadays almost all the gadgets are equipped with wireless features and due to lack of continuous connectivity in wireless networks data transmission always remains a tedious task. Currently most of the intranet networks are adhoc as they are not relayed through centric data transmission system. This gives rise to lack of connectivity in the network. Due to this nature of network DTN (Disruption Tolerant network) concept arises where AODV and DSR routing protocols are failed to perform well. This kind of fast and daisy networks attract two phase commit protocol that has proved to be one of the best solution as of date where it holds the properties of commit or abort the transaction in run time and even it can roll back the transaction on unavailability of the resources and hold it till the gaining of access. However, the measurement and accountability of the heterogeneous network is a tedious task.

Advancement in wireless technology and portable computing have opened new possibilities for wireless computing.[1]. Lesser requirement of hardware and ease of deployment MANET (Mobile Ad Hoc Network) have been preferred for communication.

Major open challenges in MANET observed are scalability; address configuration, Quality of service (QOS), power consumption and security. Harsh environment and dynamic nature of environment designing better routing protocols has been challenging mission. Mesh methodology has been found to be better over tree based approach in implementation of routing protocol. Hybrid approach achieves moderate result with multicasting. Routing algorithms have been classified in Proactive and reactive according to their nature of operation. Designing new routing protocol based on existing research work is challenge taken up by research community. Reactive protocols have been found to function better in terms of less energy consumption, packet delivery ratio delay and throughput Active area of research and focus in this decade has been AODV (Ad hoc on Demand Distance Vector ) and DSR (Dynamic Source Routing) as numerous research observations present them to be effective and future scope for work. This research manuscript investigates routing protocols and focuses on designing better $2 \mathrm{pc}$ protocol.

DSR is effective protocol used for routing in Multi-Hop MANET. It is two phase procedure with route maintenance and route discovery [2].

This two phase approach helps for better route discovery, source to destination. It is loop less routing, where intermediate nodes do not need to update. Every node controls packet and forward it based on route info [2]. In problematic scenario where destination node is unknown, destination has been found by querying all nearest neighbor nodes. 


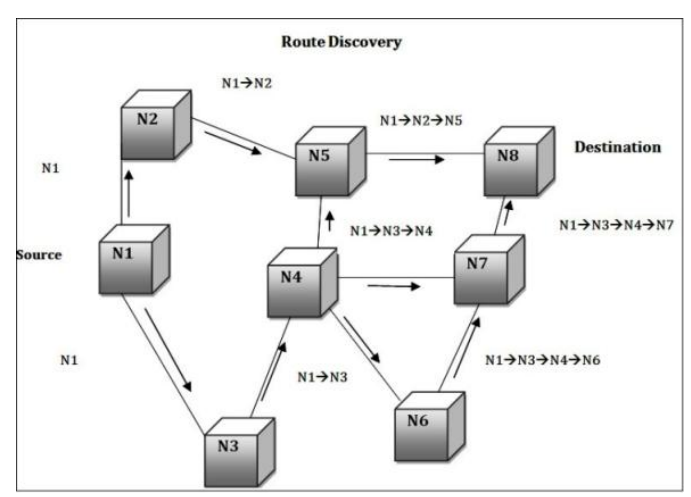

Figure 1: Route Discovery in DSR

Fig1 depicts construction of route record in DSR during route discovery. Fig.2 depicts route reply with route record

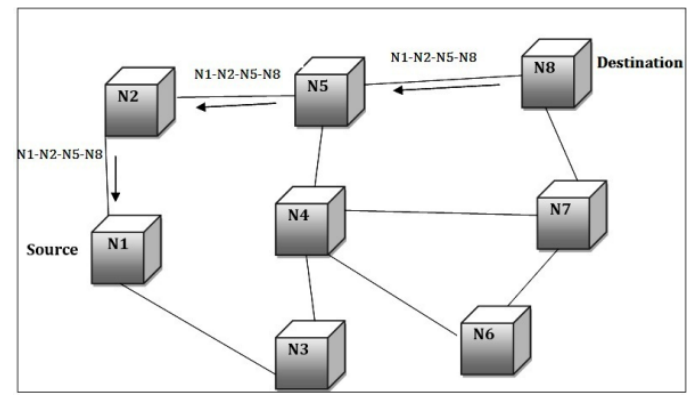

Figure 2: Route Reply in DSR

AODV is adaptive reactive routing protocol which is dynamic to link conditions and lower network utilization in MANET [2]. AODV doesn't discover and uphold any route unless nodes require to communicate. Sequence number of destination node has been used to guarantee communication. Functioning pattern of AODV is similar to DSR two phase approach. Initially to send information RREQ (route request packet) has to be sent to all neighbors and RREQ has to be acknowledged by RREP (route request reply). In AODV if reply has been received from node it means fresh route exists to destination. RERR (Route Error) control packet is being sent to avoid break in linkage.

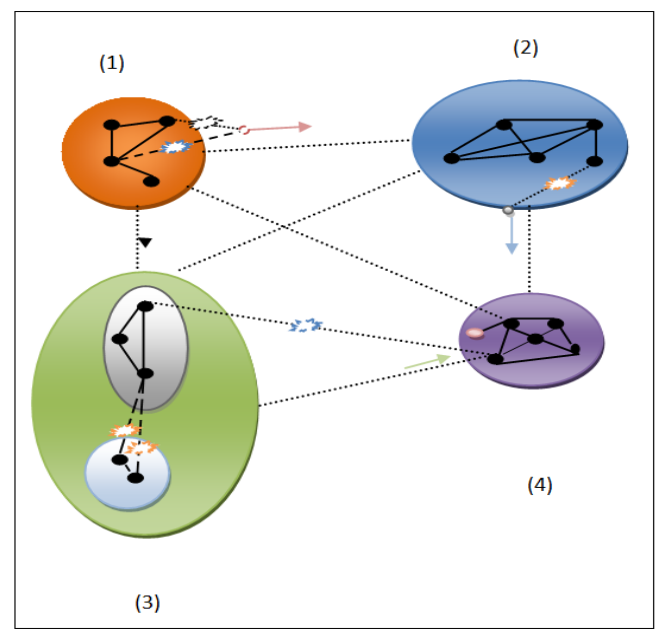

Figure 3: interconnection of regional networks through an overlay DTN architecture.

Legend of Figure 3:

$\| \rightarrow$ On Way from region(1) to (2) $\| \rightarrow$ Leaving Region

(2). $\| \rightarrow$ Arriving region (4). $\| \rightarrow$ Moving in same region. $\| \ldots .$.
Connection in nodes. $\|$ disrupted link.||---- Interconnection in regions.

In wireless communication distinct changes occur in single operation and need atomic commit to ensure successful operation. In order to maintain consistent state and overcome unreliability of networks atomic commit protocols are necessary.

Current scenario ICNs (Intermittently Connected Networks) have to be deployed in numerous extreme situations, suffering from link disruption and affect heterogonous nature of ICNs. DTN (Delay-/Disruption-Tolerant Network) Networking has appeared as extremely lively part of research in networking, where scholars compete to solve problems of ICNs [3]. Delay-Disruption-Tolerant Networking is design architecture for ICN's developed by Engineering Task Force [3]. It operates above protocol stack of different ICNs to enable functionality of gateway through storage capacity, numerous protocol techniques, parallel forwarding, and replication other network impairments like error forwarding and correction. DTN acts a layer transitorily connecting isolated network with new addressing scheme, encapsulating IPs and persistent storage for better network.

DTN is Encouraging Solution for better wireless communication with minimized latency and maximum delivery ratio. Observation in DTN [3] are message duplication increased delivery ratio and decreased delay time. Open research challenge in DTN implementation is power consumption (battery depletion) and lower buffer space. If these issues are handled with addition techniques a scalable network system would be achieved.

2PC (Two phase Commit) protocol is Atomic commit protocol (ACP) most commonly used in distributed networking and transaction [4]. It is Distributed procedure that co-ordinates every process (nodes in MANET) in system whether to commit or abort. Two phase commit protocol is 2 step procedures, initially voting phase is initiated to initiate all nodes participating in communication to vote yes or no. In second step if all node commit yes then only action is committed. This approach grantees better communication but still open research challenges exist in two PC protocol. Subsequent part reviews manuscripts on networking domain.

AI-Houmaily et.al.[5] introduced 1-2PC protocol to commit transaction in network. Large message and log complexity was observed in $2 \mathrm{PC}$ protocol. 1PC assumes implicit yes-no specification suffering forward recovery. Proposed Algorithm is adaptive and selects two phase or one phase commit according to system requirement. This algorithm has been found better in limited bandwidth and deferred consistency. This technique has been tested on high volume of small transactions only. However, it could have been evaluated for higher volume of large transactions.

eFranks et.al. [6] reported SIP protocol with layered networking queue model for standalone and cluster configuration. SIP (Session Initiation Protocol) protocol has been used here to establish session in between two parties. Java API has been used for developing SIP application . A Two Phase Methodology with fixed rate server for modeling Ethernet was presented and found to be very good but the Bottleneck is limited by the computation on SIP servers only that should have been determined whether caused by hardware or Ethernet or buffers scarcity.

Reinke Christomph et.al. [7] Have reported concurrency control protocol in WSN (Wireless Sensor Networks) in 
which Transaction processing ability is required to grantee data consistency at runtime. Research work compares S2PL (Strict-Two-Phase Locking), TO (Timestamp Ordering), FOCC (Forward Oriented Optimistic Concurrency Control). $\mathrm{S} 2 \mathrm{Pl}$ is found to have very good commit rate and lesser cost than other. Here, $2 \mathrm{pc}$ network with locking facility has been implemented and showed better performance in constrained environment. However this research work has been implemented at simulation level only and no practical implementation have been reported to the best of our knowledge.

Congiu and Teresa et.al.[8,9] Address research problem of managing distributed namespace for operations like RENAME, CREATE, DELETE. Protocols like 2PC with locking mechanism are costly and require higher message exchange. One phase commit protocol has been used for inter metadata messaging but this work also has been simulated and not tested in real time. Performance measure of One PC protocol is observed is $50 \%$ better than $2 \mathrm{PC}$ which contradicts other research scholar's evaluations

Chauhan et.al.[10] have reported Optimized commit protocols also where O-2PC has been implemented on base 2PC on delayed and instantly consistency constraints by overriding voting stage where commencement of protocol is done from member side comparative results on $2 \mathrm{pc} 3 \mathrm{pc}$ protocols are simulated and presented. But this work is implemented in java and still exists at simulation level and not tested on real time scenario

Abuya et.al [11] reported two phases Commit protocol which is a very good measure for safekeeping ACID (Atomicity Consistency Isolation Durability) properties in transactions. 2 PC ensures every single transaction is executed. Proposed System with $2 \mathrm{pc}$ Communication protocol ensures all transactions are executed with overhead reduction. Proposed algorithm would enhance back ends of applications like Mysql, Oracle.

Sanjeev Kumar [12] has reported enhanced 2 pc protocol that eliminates the blocking problem. Three Pc protocol has been found here to overcome this issue, but additional message overhead reduces system performance. So we propose here algorithm E2PC (Extended Two phase commit) which is enhanced version of two pc only with decision control to overcome blocking issue. The technique has been tested on real time basis and has been found to be good for web portal with no communication failure.

Sun and Fang [13] have reported solution of the issue of authentication when authentication server is down. Selfauthentication i.e. local authentication has been facilitated and session key for two party communication. CMFC (Chaotic Maps-based Scheme against Fragile Communication) framework has been implemented which achieves non failure in security authentication with $2 \mathrm{pc}$ protocol

Johnson et.al. [14] have reported the high concurrency in transaction processing. Here, multicore hardware requires special software to achieve parallelism as the transaction process needs to co-ordinate communication between independent agents. Achieving this is very difficult for transaction process. Unbound fixed cooperative communication is required, with scalability and reliability which can be achieved with $2 \mathrm{p}$ protocol and results have not reported so far.
By studying details merits, demerits and scope of all the techniques and proper analysis we found that address configuration, locking mechanism, Quality of service (QOS), power consumption (battery depletion), security and higher buffer space requirement is still challenging problem for designing a perfect and suitable protocol for better communication. Also most of the Research work has been implemented in simulation and not tested on real time scenario which is major drawback. Therefore a better methodology with real time testing is the inevitable requirement to overcome demerits of existing techniques. Two PC protocol has been found to be better compared to other protocols and incurs lesser overhead. Hence focus of research remains in designing scalable methodology for implementing two pc protocol. Therefore, we propose a modified methodology which is tested in real time environment and reported for the first time in this paper.

This paper is further classified as section 2 for proposed methodology techniques of our system, Section 3 for Result and discussions where results of our technique using to phase commit protocols have been compared with the results of the other routing protocols. Finally section 4 concludes the paper.

\section{PROPOSED METHODOLOGY}

This section narrates about the techniques that are followed during the implementation of two phase commit protocol in the wireless LAN (Local Area Network) with below mentioned steps as depicted in figure 4 .

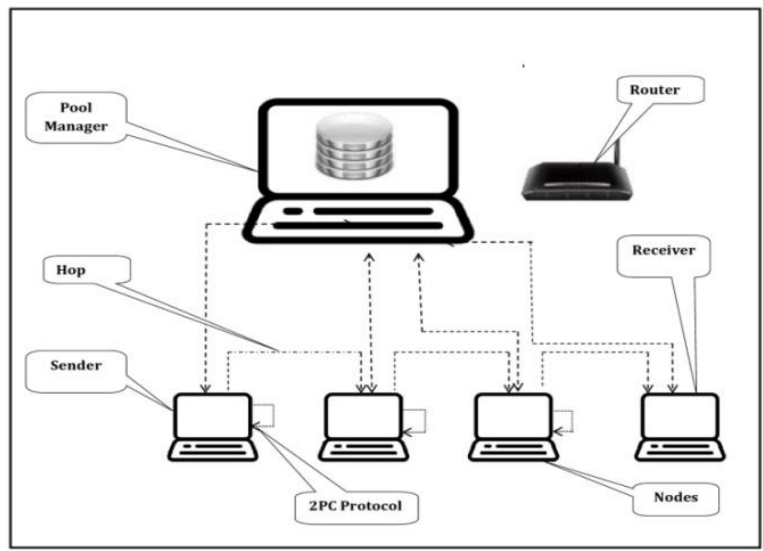

Figure 4: System Overview of Two phase commit protocol in Wireless LAN

Step 1: Network Setup- Here in this very first step of our system a network has been setup by activating every node in the network for pool manager as its monitor and this constitutes a network pool. The System is designed in such a way that on activating each node it will register its machine name with its IP in the database that has been maintained by the pool manager. The table eventually provides the routing table information for two phase commit protocol as narrating in the successive steps.

Step 2: Routing initialization- Here a sender node will select its receiver from the all available receiver's details on its user interface which eventually shares the database of the pool manager. As soon as the sender node sends the data to the receiver node proposed system shares the database of the pool manager and sets the sender node as the beginning node and receiver node as the end node of routing process. 
Step 3: Co-operative Pool manager - Here in this process every instance sender node will contact the pool manager for the routing info. Once the routing info is provided by the pool manager to the instance sender node then data will be delivered using two phase commit protocol technique as explained in the next step.

Step 4: Two phase commit routing protocol - As soon as sender instance node receives the routing info from the pool manager, then instance sender node sends knock knock message to the receiver node for its availability in the network pool and keeps waiting for its reply from the receiver node.

Two phase commit protocols here can take the decision of roll backing the process or discard on its threshold timing. Due to this characteristics of the two phase commit protocol the transmission of the data can be withhold on unavailability of the network to ensure the data delivery in proper style.

The whole process can be depicted with the following algorithm 1 .

\begin{tabular}{l} 
Algorithm 1: Two Phase Commit Protocol \\
\hline // Input: Sender Data $\mathbf{D}$ \\
// Destination Node $\mathbf{D}_{\mathbf{n}}$ \\
// Output: Data Delivery to $\mathbf{D}_{\mathbf{n}}$ through Successful 2PC \\
Routing Protocol \\
Step 0: Start \\
Step 1: Add all the nodes $\mathrm{N}_{\mathrm{i}}$ under Pool managers $\mathbf{P}_{\mathbf{m}}$ \\
into pool $\mathbf{P}$ \\
Step 2: Activate All the nodes \\
Step 3: Select Data $\mathbf{D}$ by source node $\mathbf{S}_{\mathbf{n}}$ \\
Step 4: Select Destination node $\mathbf{D}_{\mathbf{n}}$ \\
Step 5: WHILE D $\mathbb{C}$ to $\mathbf{D}_{\mathbf{n}}$ \\
Step 6: FOR each HOP $\mathrm{H}_{\mathrm{i}}$ \\
Step 7: WHILE $\mathbf{S}_{\mathbf{n i r}}$ !=NULL $\left(\mathbf{S}_{\mathbf{n i r}}\right.$ is Source node \\
request replay for the instance) \\
Step 8: Transfer Data to $\mathrm{D}_{\mathrm{i}}\left(\mathrm{D}_{\mathrm{i}}\right.$ is Destination node for \\
the instance) \\
Step 9: END WHILE \\
Step 10: END FOR \\
Step 11: END WHILE \\
Step 12: Stop
\end{tabular}

\section{RESULTS AND DISCUSSIONS}

The proposed system uses twenty one machines of standard configuration with core i3 processor and 4 GB RAM for the experimental process of two phase commit protocol. One machine out of Twenty one Machines is assigned the role of Pool manager which is allocated to maintain the database and rest machines are given the role of the nodes.

All the machines are equipped with Windows operating system and all are having java supporting features which are using

Net beans as the development IDE (Integrated Development Environment). System is put under hammer for number of tests for the proper deployment of two phase commit protocol in disruption tolerant network scenario which uses D- Link two Antenna wireless routers.
Table 1: Hopping time performance in Time

\begin{tabular}{|r|r|r|r|}
\hline $\begin{array}{r}\text { No of } \\
\text { Hops }\end{array}$ & $\begin{array}{c}\text { No of } \\
\text { Packets }\end{array}$ & $\begin{array}{c}\text { Average Transmission Time } \\
\text { (in Milliseconds) } \\
\text { Two Phase Commit Protocol }\end{array}$ & $\begin{array}{c}\text { Average Transmission Time } \\
\text { (in Milliseconds) } \\
\text { DSDV Protocol }\end{array}$ \\
\hline 1 & 5334 & 675.64 & 1000 \\
\hline 2 & 5334 & 728.98 & 1000 \\
\hline 3 & 5334 & 1262.38 & 1000 \\
\hline 4 & 5334 & 1369.06 & 1000 \\
\hline 5 & 5334 & 1102.36 & 1000 \\
\hline 6 & 5334 & 693.42 & 1000 \\
\hline 7 & 5334 & 728.98 & 1000 \\
\hline 8 & 5334 & 746.76 & 1000 \\
\hline 9 & 5334 & 657.86 & 1000 \\
\hline 10 & 5334 & 497.84 & 1000 \\
\hline 11 & 5334 & 462.28 & 1000 \\
\hline 12 & 5334 & 746.76 & 1000 \\
\hline 13 & 5334 & 746.76 & 1000 \\
\hline 14 & 5334 & 835.66 & 1000 \\
\hline 15 & 5334 & 1173.48 & 1000 \\
\hline 16 & 5334 & 1209.04 & 1000 \\
\hline 17 & 5334 & 728.98 & 1000 \\
\hline 18 & 5334 & 728.98 & 1000 \\
\hline 19 & 5334 & 675.64 & 1000 \\
\hline & & & \\
\hline
\end{tabular}

Experiments are conducted in our system for the data transmission among the twenty nodes with two phase commit protocol, which records the successful transmission with delay time recoded in the table 1 . In table 1 Number of packets sent is normalized for 5334 packets according to table 3 of [15] for 300 packets of sent data from our experiment. And whereas the [15] deals with the DSDV (Destination-Sequenced Distance-Vector Routing) routing protocol in wireless network for detection of the gray hole.

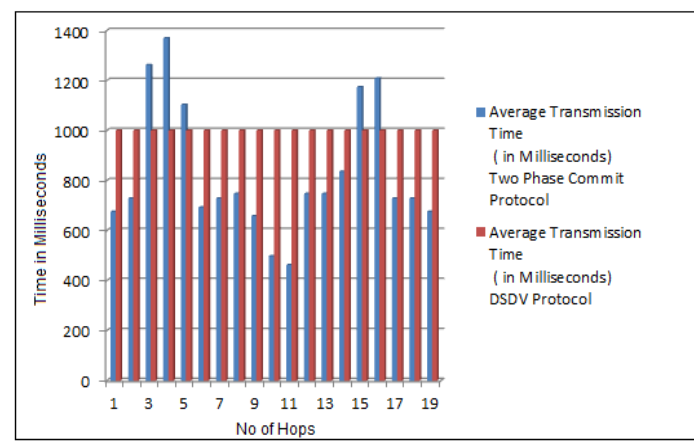

Figure 5: Hopping time comparison graph

When the values of the table 1 are drawn in a plot as shown in figure 5 . We found the fact that system proposed by this paper for data transmission using two phase commits protocol over DTN are providing far better results than that of system proposed by [15]. In the plot observed another fact that two phase commit protocol takes average of 830 milliseconds of time for each hop, whereas the DSDV protocol takes average time of 1000 milliseconds for each hop. 
One interesting thing draw from this table 1 that two phase commit protocol takes almost 15770 milliseconds of time for total transmission of the data using 20 nodes, whereas the DSDV protocol of [15] takes almost 20000 milliseconds. This concludes that decision of considering two phase commit protocol over the DTN is a right choice and it always perform in good range.

The Average end to end delay of the proposed methodology is measured and compared with other technique of iETT as mentioned in [16]. iETT is an improved version of the ETT (Expected transmission Time), a routing sequence selection method - which selects the routing path based on the availability of the wireless links and other link quality conditions of the network nodes. Whereas proposed system applies and analyzes the availability of the network link by incorporating two phases commit protocol which is having excellent hold on the transmission control. The observed end to end delay is recorded in the table 2 .

Table 2: Average Latency (in Second)

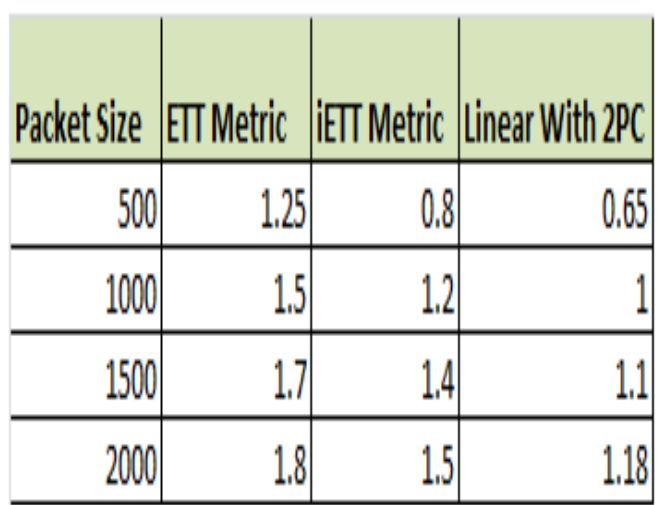

On drawing the plot for above facts we got the comparative structure as mentioned in figure 6 .



Figure 6: Average Latency (Seconds)

On observing and analyzing above plot some facts are revealed like - Even though on use of linear path selection process in our implementation two phase commit protocol having excellent hold on transmission mode to select the Strong link wireless nodes. Due to this the end to end delay can be diminished reasonably as shown in the figure 6 . This proves the quality of rules and analysis power of two phase commit protocol exceeds in many scenarios of routing in wireless paradigm.

\section{CONCLUSION AND FUTURE SCOPE}

The wide spread use of wireless network forces us to invent advance routing protocols where data need to be deliver in high accuracy. But due to increasing size of digital gadgets and unavailability of the network for moving wireless devices this task always lives in doubt. Therefore, this problem is addressed with the tag of Disruption tolerant network (DTN), which opens many options to deal with this paradigm. This paper analyze and accumulates important factors of AODV and DSR protocols on DTN networks to come to know the flaws of the protocols.

As an answer to this two phase commit protocol on deployment in real time scenario has been analyzed for the DTN networks and. we found most astonishing results in the improvement of the routing paradigm as compared to DSR and AODV protocols for routing throughput and for average end to end delay. It shows that this paper successfully incorporates the characteristics of two phase commit protocols in real time in DTN wireless network.

As a future scope, this paper is having perception of deploying two phase commit protocol in real time wireless hybrid network to achieve fast vertical handoff in between the different network pools also in future it will considered and resolved the problem related to protocols.

\section{ACKNOWLEDGEMENT}

The authors wish to express their appreciation for the technical support of International Accreditation Council of Quality Education and Research (IACQER) www.iacqer.com and International R\&D center for cloud computing and data mining (www.c3dmw.com) for the research infrastructure. One of the author (GUM) thanks to the parents and wife M/S Seema for the moral support and daughters Gayatri and Dheera for their long hours patience during the experimentation.

\section{REFERENCES}

[1] De Morais Cordeiro, Carlos, Hrishikesh Gossain, and Dbarma P. Agrawal. 2003. Multicast over wireless mobile ad hoc networks present and future directions. IEEE network 17.1 (2003): 52-59.

[2] Barati, Mehdi, et al. 2012. Performance evaluation of energy consumption for AODV and DSR routing protocols in MANET. Computer \& Information Science (ICCIS), 2012 International Conference on. Vol. 2. IEEE, (2012).

[3] Maurice J. Khabbaz, Chadi M. Assi, and Wissam F. Fawaz, 2011. Disruption-Tolerant Networking A Comprehensive Survey on Recent Developments And Persisting Challenges. IEEE communications surveys \& tutorials, accepted for publication DOI:10.1109/SURV.2011.041911.00093.

[4] https://en.wikipedia.org/wiki/Two-phase.

[5] Al-Houmaily, Yousef J., and Panos K. Chrysanthis. 2004. 1-2PC: the one-two phase atomic commit protocol Proceedings of the ACM symposium on Applied computing. ACM.(2004).

[6] eFranks, Greg, Danny Lau, and Curtis Hrischuk. 2011 Performance measurements and modeling of a javabased session initiation protocol (SIP) application server. Proceedings of the joint ACM SIGSOFT 
conference--QoSA and ACM SIGSOFT symposium-ISARCS on Quality of software architectures--QoSA and architecting critical systems--ISARCS. ACM.(2011).

[7] Reinke, Christoph, et al.2011. Analysis and comparison of concurrency control protocols for wireless sensor networks. International Conference on Distributed Computing in Sensor Systems and Workshops (DCOSS). IEEE, (2011).

[8] Congiu, Giuseppe, et al. 2012. One Phase Commit: A Low Overhead Atomic Commitment Protocol for Scalable Metadata Services. CLUSTER Workshops.( 2012).

[9] Teresa K. Abuya ,Dr.Richard M. Rimiru, and Cheruiyot W.K. 2014. An Improved Failure recovery algorithm in two phase commit protocol for transcation atomicity, Volume 5, No. 12, JGRCS, (2014).

[10] Chauhan, Ganpat Singh, Mukesh Kumar Gupta, and Ajay Khunteta.. 2014. IOCP: Implementation of Optimized Commit Protocol. Recent Advances and Innovations in Engineering ICRAIE, (2014).

[11] Abuya, Teresa K. 2015. An Improved Failure Recovery Algorithm in Two-Phase Commit Protocol for
Transaction Atomicity. Journal of Global Research in Computer Science 5.12 (2015): 01-11.

[12] Sanjeev Kumar Singh Kushwaha.2015. Extended Two Phase Commit Protocol in Real Time Distributed Database System. International Journal Of Engineering And Computer Science ISSN:2319-7242Volume 4 Issue 5 May 2015, Page No. 12188 -12193

[13] Yang Sun, Xueshuai Feng. 2016. Two-phase Commit with Security Services: Using Distinctive Proofs to Relieve Fragile Communications. International Journal of Network Security, Vol.19, No.2, PP.217-228. (Apr. 2016).

[14] Johnson, Ryan, Ippokratis Pandis, and Anastasia Ailamaki. 2014. Eliminating unscalable communication in transaction processing. The VLDB Journal 23.1 (2014): 1-23.

[15] S D Khatawkar and Nitin Trivedi. 2015. Detection of Gray hole in MANET through Cluster Analysis. 2nd International Conference on Computing for Sustainable Global Development. (IEEE 2015)

[16] Saad Biaz, Bing Qi and Yiming Ji. 2008. Improving Expected Transmission Time Metric in Multi-rate Multi-hop Networks. IEEE CCNC. (2008) 